\title{
Hidrofluoralcano como propelente dos aerossóis pressurizados de dose medida: histórico, deposição pulmonar, farmacocinética, eficácia e segurança
}

\author{
Hydrofluoroalkane as a propellant for pressurized metered-dose inhalers: \\ history, pulmonary deposition, pharmacokinetics, efficacy and safety
}

\author{
Cássio C. Ibiapina1, Álvaro A. Cruz², Paulo A. M. Camargos ${ }^{3}$
}

\section{Resumo}

Objetivo: Rever a literatura sobre o hidrofluoralcano como propelente dos inaladores de dose medida contendo medicamentos empregados na asma.

Fontes dos dados: O levantamento bibliográfico foi realizado em bancos de dados eletrônicos - MEDLINE, MDConsult, HighWire, Medscape e LILACS - e por pesquisa direta - referentes aos últimos 15 anos -, utilizando-se as seguintes palavras-chaves: hidrofluoralcano, asma e infância.

Síntese dos dados: Foram selecionados 43 artigos originais abordando a questão da substituição do clorofluorcarbono pelo hidrofluoralcano. Este gás mostrou-se como uma alternativa de propelente segura, com deposição pulmonar de 50 a $60 \%$ e eficácia significativa quando comparado com placebo $(p \leq 0,003)$ através de estudos clínicos controlados. A maioria das pesquisas realizadas com o hidrofluoralcano emprega o dipropionato de beclometasona. O custo anual aproximado do tratamento com dipropionato de beclometasona/hidrofluoralcano foi menor do que com dipropionato de beclometasona/clorofluorcarbono. Alguns estudos avaliaram o salbutamol, a fluticasona, a flunisolida e a associação fluticasona-salmeterol tendo o hidrofluoralcano como propelente em inaladores pressurizados de dose medida.

Conclusões: Ficou evidenciada em adultos a eficácia e segurança do hidrofluoralcano como propelente de broncodilatadores e glicocorticosteróides em aerossol, bem como a melhor deposição pulmonar das partículas, de um modo geral. Todavia, são escassos os dados na literatura acerca do uso do hidrofluoralcano na faixa etária pediátrica. Seria importante conduzir estudos adicionais em crianças e adolescentes.

J Pediatr (Rio J). 2004;80(6):441-6: Asma, hidrofluoralcano, tratamento, aerossol.

1. Doutorando do Programa de Pós-graduação em Pediatria, Universidade Federal de Minas Gerais (UFMG), Belo Horizonte, MG.

2. Coordenador do Centro de Enfermidades Respiratórias, Faculdade de Medicina, Universidade Federal da Bahia (UFBA), Salvador, BA.

3. Professor titular, Departamento de Pediatria, Faculdade de Medicina e Chefe da Unidade de Pneumologia Pediátrica, Hospital das Clínicas, UFMG, Belo Horizonte, MG.

Artigo submetido em 22.03.04, aceito em 31.03.04.

Como citar este artigo: Ibiapina CC, Cruz AA, Camargos PAM. Hidrofluoralcano como propelente dos aerossóis pressurizados de dose medida: histórico, deposição pulmonar, farmacocinética, eficácia e segurança. J Pediatr (Rio J). 2004;80:441-6.

\begin{abstract}
Objective: To review the literature about hydrofluoroalkane as a propellent of pressurized metered-dose inhalers containing antiasthma drugs.

Sources of data: Bibliographic search in electronic databases (MEDLINE, MDConsult, HighWire, Medscape and LILACS) and direct search referring to the past 15 years, using the key words hydrofluoroalkane, asthma and childhood were carried out.

Summary of the findings: 43 original articles on the replacement of clorofluorcarbon by hydrofluoralkane were selected. Hydrofluoralkane showed to be a safe propellent, with pulmonary deposition ranging from 50 to $60 \%$, and to have significant efficacy, when compared with placebo $(p \leq 0.003)$ in controlled clinical trials. Most works using hydrofluoralkane included beclomethasone diproprionate. Approximate annual cost of a treatment with beclomethasone diproprionate/hydrofluoralkane was lower than with beclomethasone diproprionate/clorofluorcarbon. Some studies assessed salbutamol, fluticasone, flunisolide and the association fluticasone-salmeterol, with hydrofluoralkane as propellent in pressurized metered-dose inhalers.

Conclusions: Efficacy and safety of hydrofluoralkane as propellent of bronchodilators and inhaled corticosteroids in adults was evidenced, as well as, in general, there was a better pulmonary deposition of particles. However, literature data on the use of hydrofluoralkane in the paediatric age group are still scarce and further studies with children and adolescents would be of great importance.
\end{abstract}

J Pediatr (Rio J). 2004;80(6):441-6: Asthma, hydrofluoralkane, treatment, aerosol.

\section{Introdução}

A via inalatória, utilizada desde os primórdios da história da medicina, tornou-se popular no final do século 19. Naquela época, adicionavam-se medicamentos em água fervente para que os pacientes pudessem inalar seu vapor ${ }^{1}$.

$\mathrm{Na}$ literatura especializada do século 20 , os primeiros relatos da moderna era da aerossolterapia em asma datam da década de 50, quando foram apresentados os inaladores pressurizados de dose medida (IPDM), que representaram inegável avanço no tratamento das doenças respiratórias. $\mathrm{Na}$ atualidade, sabe-se que as prescrições de IPDM em todo o mundo excedem 500 milhões de unidades por ano, e seu uso vem aumentando a cada década². 
É reconhecido que os corticóides inalatórios constituem a terapia de primeira linha, recomendada em todos os consensos, para o controle da asma persistente, e são comumente administrados por intermédio dos IPDM. O clorofluorcarbono (CFC) é o propelente mais utilizado nos IPDM; é de baixo custo, seguro e eficaz, mas teve seu uso restringido universalmente devido a seus efeitos deletérios na camada de ozônio ${ }^{3}$.

Nos anos 70, foram registradas as primeiras evidências de que o CFC e outras substâncias contendo cloro contribuem para a depleção da camada de ozônio na estratosfera ${ }^{4}$. Com isso, a radiação ultravioleta que alcança a superfície da Terra aumenta, produzindo efeitos adversos graves no homem, como câncer de pele, catarata e diminuição da resistência imunológica. Exerce também influência sobre as aves, a vida marinha, sobre os plásticos e outros materiais ${ }^{5}$.

A zona de depleção na camada de ozônio sobre a Antártida, observada no início dos anos 80 , vem aumentando anualmente. Estudos recentes estimam seu tamanho em 23 milhões de $\mathrm{km}^{2}$, o que equivale a quase três territórios brasileiros.

O acordo internacional de entidades ambientais - o chamado Protocolo de Montreal $^{5}$-, que visa combater a produção de substâncias que interferem na camada de ozônio, foi assinado em setembro de 1987 . O acordo estabeleceu uma diminuição da produção de CFC em $50 \%$ ao ano, até 1998. Em 1990, com o aumento das evidências da relação entre o CFC e a camada de ozônio, uma emenda proibiu a fabricação de extintores de incêndio contendo CFC a partir 2000. Em 1992, com a constatação da aceleração da depleção na camada de ozônio, a produção do CFC foi banida até o final de"1995, sendo permitida apenas em medicamentos até 2005. Assim, os IPDM constituem a única exceção à produção e comercialização do CFC, por terem sido considerados essenciais no tratamento da asma6.

Em 1995, dois propelentes seguros e eficazes - hidrofluoralcano 134a (HFA 134 a) e hidrofluoralcano 227ea (HFA 227ea) - foram reconhecidos pela União Européia 6 . Em 1996, a agência americana Food and Drug Administration (FDA) aprovou o uso de HFA 134a em inaladores?.

O HFA não afeta a camada de ozônio, porém tem efeito sobre o aquecimento global, sendo um dos seis gases do efeito estufa 8 .

Em 1997, foi realizada a Convenção das Nações Unidas sobre Mudanças Climáticas - o Protocolo de Kyoto -, que aprovou as resoluções da Convenção do Meio Ambiente de 1992, realizada no Rio de Janeiro - a ECO 92. O Protocolo de Kyoto ampliou aquelas resoluções, dando ênfase à emissão de gases do efeito estufa nos Estados Unidos. É importante salientar que, apesar de o Protocolo de Kyoto ter sido ratificado por 55 nações, incluindo países desenvolvidos, não o foi pelos Estados Unidos e, por isso, não tem efeito de lei em seu território 4,6 .

O Protocolo de Kyoto criou mecanismos para o combate às alterações climáticas, visando, principalmente, à redução dos gases que contribuem para o aquecimento global. Os seis gases do efeito estufa (dióxido de carbono, metano, óxido nítrico, hidrofluoralcano, perfluorcarbono e hexafluorido sul- foroso) foram reunidos em um único grupo. Com essa abordagem, os países têm a flexibilidade de escolher a percentagem de redução de cada gás para atingir o objetivo de diminuição total fixado no protocolo ${ }^{6}$.

O HFA, juntamente com o hexafluorido sulforoso e os perfluorcarbonos, são grandes geradores de calor. No entanto, como constituem apenas $1,8 \%$ dos gases do efeito estufa emitidos nos Estados Unidos, contribuem pouco para o aquecimento global 6,7 .

A aplicação do HFA em IPDM é uma opção de tratamento médico de inegável valor para a saúde; por isso, todas as nações devem assegurar sua disponibilização para a população. Nessa fase de transição do uso do CFC para o do HFA, este artigo de revisão tem como objetivo apresentar aspectos relacionados a características farmacocinéticas, de eficácia, segurança, bem como as implicações dessa mudança na abordagem do paciente asmático.

\section{Deposição do dipropionato de beclometasona e IPDM contendo CFC e HFA}

O processo de transição do uso do propelente CFC para o HFA propiciou o desenvolvimento de tecnologia de produção de aerossol. Dipropionato de beclometasona (DPB), o corticóide inalatório mais antigo, vem sendo usado na terapêutica da asma há mais de duas décadas. A associação do DPB com HFA 134a resulta em aerossol dotado de partículas muito menores do que as geradas pelos inaladores que usam o CFC. Modelos matemáticos que relacionam o tamanho da partícula com o local de deposição no trato respiratório e estudos com modelos experimentais de vias aéreas superiores demonstram que as partículas geradas com o HFA se depositam em maior quantidade nas pequenas vias aéreas, enquanto aquelas geradas pelo CFC tendem a depositar-se mais proximalmente, inclusive na orofaringe ${ }^{9-13}$.

Os IPDM com CFC e os inaladores de pó liberam a droga, que se deposita, primariamente, na orofaringe, e secundariamente, nas grandes vias aéreas. Do DPB aplicado através de IPDM com CFC, mais de $90 \%$ da droga são depositados na orofaringe e menos de $10 \%$ nos pulmões. Se aplicado com HFA, a taxa de deposição pulmonar pode chegar a $60 \%$, enquanto cerca de $30 \%$ se depositam na orofaringe ${ }^{10}$. O tamanho médio da partícula do HFA-DPB é 1,1 micra, e a do CFC-DPB, 3,5 micra. A força manual necessária para acionar o spray de HFA-DPB é três vezes menor do que a requerida para a formulação com CFC. A duração do spray HFA-DPB é mais longa (250 milissegundos) do que o CFCDPB (150 milissegundos). A temperatura do spray é maior no HFA-DPB $\left(5^{\circ} \mathrm{C}\right)$ do que no CFC-DPB $\left(-20^{\circ} \mathrm{C}\right)$, o que reduz o indesejável efeito freon. As características físicas apresentadas pelo jato e pelas partículas do composto HFA-DPB são responsáveis pela maior deposição pulmonar e menor deposição na orofaringe ${ }^{12}$.

É interessante salientar que menos de $50 \%$ dos pacientes aplicam a técnica inalatória de maneira adequada, devido ao acionamento do inalador antes do início da inspiração ou ao início tardio do ciclo inspiratório. O tamanho da partícula menor e a duração do spray maior do HFA-DPB promovem 
melhor deposição nas vias aéreas mesmo naqueles pacientes com problemas graves de coordenação ${ }^{10}$.

Em estudo clínico de dose-resposta, Bogston et al. demonstraram que o HFA-DPB tem eficácia equivalente a uma dose 2,6 vezes maior de CFC-DPB quando avaliada pelo volume expiratório forçado no primeiro segundo $\left(V_{E F}\right)$, e a uma dose 3,2 vezes menor do HFA-DPB quando o parâmetro estudado foi o valor máximo do fluxo expiratório médio $\left(\mathrm{FEF}_{25-75}\right)^{14}$.

\section{HFA como propelente de outras drogas: salbutamol, fluticasona, flunisolida, fluticasona associada com salmeterol e a apresentação não- extrafina}

Na literatura especializada, vários estudos demonstraram que, em formulações com salbutamol, o tamanho da partícula emitida pelo IPDM-CFC, pelo IPDM-HFA e pela formulação em pó foram equivalentes. Pode-se inferir que a percentagem de deposição pulmonar e, portanto, os efeitos clínicos são semelhantes para as três formulações. Esses estudos demonstraram, ainda, não haver diferença significativa na melhora do $\operatorname{VEF}_{1}{ }^{14-23}$.

Dois estudos avaliaram a eficácia da fluticasona-HFA. Fowler et al., em um estudo randomizado, duplo-cego, estudando 18 pacientes adultos durante 6 semanas, compararam a eficácia da fluticasona-HFA em relação à fluticasona-CFC. Um grupo composto de nove pacientes fez uso de $500 \mu \mathrm{g}$ de fluticasona-HFA, e o outro utilizou uma dose de $1.000 \mu \mathrm{g}$ de fluticasona-CFC, não sendo verificada diferença estaticamente significativa $(p=0,21)$ entre os grupos ${ }^{24}$. Langley et al. publicaram um estudo onde apresentam dados demonstrando redução da hiper-responsividade da via aérea de forma equivalente quando se utiliza fluticasona-HFA e fluticasona pelo sistema Diskhaler ${ }^{\circledR}$ em doses iguais ${ }^{25}$.

No Brasil, até o presente momento, o salbutamol e a fluticasona, ambos formulados isoladamente, e a associação fluticasona-salmeterol são as opções disponíveis no mercado. Entretanto, espera-se que apresentações de outras drogas em formulações com HFA sejam lançadas futuramente em nosso país, em virtude da iminente expiração do prazo de comercialização dos IPDM contendo CFC.

Hauber et al. publicaram um ensaio clínico realizado em 12 asmáticos adultos onde demonstram a eficácia da flunisolida-HFA ${ }^{26}$. Os autores constataram supressão da inflamação eosinofílica em vias aéreas periféricas e centrais através da análise de biópsias trans- e endobrônquicas, antes e após 0 uso de flunisolida-HFA. Encontraram melhora significativa da função pulmonar ( $p=0,012$ ), diminuição do número de eosinófilos, com uma diminuição de $51,5 \pm 5$ para $14,6 \pm 3,2$ células $/ \mathrm{mm}^{2}$, interleucina- 5 e eotaxina em vias centrais e periféricas com uma redução de $37,3 \pm 6,2$ para $16,7 \pm 3,2 / \mathrm{mm}^{2}$ e $38,8 \pm 5,5$ para $22,3 \pm 3,1 / \mathrm{mm}^{2}$, respectivamente. Nesse estudo, os pesquisadores destacam o possível papel do menor diâmetro das partículas de medicação obtidas com o HFA, permitindo a chegada do corticóide às vias aéreas periféricas, o que também foi demonstrado em outros estudos ${ }^{26-28}$.

Currie et al., analisando 20 asmáticos adultos em um estudo randomizado duplo-cego, observaram que a fluticasona-HFA e sua apresentação associada com salmeterol foram eficazes em reduzir a PD20 (dose que causou queda de $20 \%$ no $\mathrm{VEF}_{1}$ ), mas somente a combinação fluticasona-salmeterol determinou melhora no $\mathrm{VEF}_{1}, \mathrm{FEF}_{25-75}$ e peak flow matinal, concluindo que a combinação confere melhora na hiperresponsividade brônquica e no calibre das vias aéreas, enquanto o corticóide isolado atua somente naquele primeiro parâmetro. A combinação fluticasona-salmeterol pode resultar em maior deposição periférica do corticóide no pulmão, o que melhora a atividade antiinflamatória do mesmo nas pequenas vias aéreas ${ }^{29}$.

Outra alternativa existente é a formulação HFA nãoextrafina, que foi registrada com a denominação Modulite ${ }^{\circledR}$. O Modulite ${ }^{\circledR}$ também apresenta como propelente o HFA 134a, mas neste caso foi adicionado um solvente não-volátil, o glicerol, que gera partículas maiores, ou seja, com tamanho mais próximo ao das partículas de CFC. Além disso, alterouse o diâmetro do orifício de saída do jato, o que tornou a nuvem de medicação mais semelhante à do $\mathrm{CFC}^{30,31}$. Atualmente, encontra-se no mercado internacional a DPB-Modulite $^{\circledR}$ e a budesonida-Modulite ${ }^{\circledR}$. A vantagem da tecnologia Modulite ${ }^{\circledR}$ é a maior semelhança com os IPDM que utilizam o propelente CFC. Isso permite que a transição CFC/HFA seja mais fácil de planejar, já que não há necessidade de alterar as doses da medicação usada, pois a deposição pulmonar e a absorção são semelhantes às dos IPDM com CFC porque o tamanho das partículas é semelhante ${ }^{32}$.

\section{IPDM contendo CFC e HFA: diferenças farmacocinéticas}

Gross et al. ${ }^{33}$ mostram que pacientes adultos com asma moderada que permaneciam sintomáticos com baixas doses de CFC-DPB ( $800 \mu \mathrm{g} /$ dia do corticóide) podiam ser controlados com uma dose menor de HFA-DPB (400 $\mu \mathrm{g} /$ dia do corticóide). Essa dose de HFA também mostrou benefícios significantes quando comparada com o placebo $(p \leq 0,003)$. Os autores apontam algumas vantagens do HFA-DPB em relação ao CFC-DPB, tais como: uma provável redução no custo do tratamento, pois se usa a metade da dose preconizada de CFC-DPB; menores efeitos colaterais locais (disfonia, candidíase) em decorrência da dose efetiva menor e da redução da deposição da droga na orofaringe; menores efeitos colaterais sistêmicos (supressão do eixo hipotálamohipófise-adrenal, púrpura, osteopenia); e maior deposição nas vias aéreas periféricas ${ }^{33}$.

De forma complementar, Magnussen et al. avaliaram a hiper-responsividade brônquica à histamina como parâmetro adicional de monitorização do tratamento com corticosteróides inalatórios em pacientes asmáticos. Um estudo multicêntrico, randomizado e duplo-cego acompanhou 164 pacientes adultos que receberam $1.000 \mu \mathrm{g}$ de CFC-DPB no período runin de 4 semanas. Dos 150 indivíduos admitidos para a segunda etapa, 72 foram mantidos com o mesmo tratamento e os 78 restantes passaram a receber $400 \mu \mathrm{g}$ de DPB-HFA. Não se detectaram diferenças estatisticamente significativas entre os dois grupos em relação aos sintomas, à função pulmonar, a hiper-responsividade da via aérea e aos marcadores séricos de inflamação ao final do período run-in e ao final da segunda fase 34 . 
Estudos recentes mostram que o processo inflamatório na asma ocorre tanto nas grandes quanto nas pequenas vias aéreas, além do remodelamento que acomete estas últimas $^{9,35}$. Este remodelamento pode ser o responsável pelo aumento da resistência das pequenas vias aéreas, mesmo em pacientes assintomáticos. O significado clínico do envolvimento das pequenas vias aéreas na asma, sua contribuição para um desfecho fatal ou para o rápido declínio da função pulmonar é ainda incerto, mas, se comprovado, implicaria mais uma vantagem do HFA-DPB, pois essa preparação atinge mais facilmente as vias aéreas periféricas ${ }^{9,35}$.

\section{Eficácia, segurança e tolerabilidade dos fármacos associados ao HFA}

As características físicas do spray aerossol extrafino HFADPB permitem que a medicação alcance igualmente as grandes, médias e pequenas vias aéreas. Essa distribuição da medicação possibilita a redução de sua dose nominal quando comparada com o CFC-DPB por meio de aerossol pressurizado, mantendo uma potência comparável à da fluticasonaCFC. Essa nuvem extrafina de aerossol apresenta, ainda, outra vantagem: não produz acúmulos tissulares ou séricos quando administrada com intervalos de 12 horas entre as doses. Além disso, mesmo acima da dose máxima diária recomendada $(800 \mu \mathrm{g})$, parece não provocar efeitos colaterais sistêmicos relevantes ${ }^{36}$.

Um revisão publicada recentemente na literatura nacional acerca da inflamação nas pequenas vias aéreas em asmáticos, por Cruz \& Ponte, salienta que a inflamação das pequenas vias aéreas pode trazer conseqüências clínicas importantes, como remodelamento e obstrução irreversível, sendo necessário buscar estratégias para o tratamento da inflamação nessa região do pulmão. O emprego de corticóide inalatório por meio de IPDM tendo o HFA como propelente seria uma alternativa para tal fim ${ }^{37}$.

A Tabela 1 reúne os principais estudos identificados na revisão bibliográfica acerca da eficácia dos IPDM tendo como propelente o HFA, e nela são apresentados aspectos relevantes como autor e país, ano de publicação, droga estudada, delineamento do estudo, tamanho da amostra e faixa etária.

Os trabalhos relacionados na tabela apresentam elementos acerca da eficácia dos fármacos associados ao HFA, e verifica-se que a maioria das pesquisas realizadas com o HFA emprega o DPB. Alguns estudos avaliam o salbutamol, a fluticasona, a flunisolida e a associação fluticasona-salmeterol. Verifica-se, ainda, a existência de apenas dois estudos que avaliaram exclusivamente a faixa etária pediátrica.

O HFA-DPB foi exaustivamente testado para avaliar sua segurança e sua tolerabilidade. Não eram esperados eventos adversos surpreendentes, pois o DPB já vem sendo utilizado há mais de 30 anos. Algumas diferenças, no entanto, foram observadas devido à menor deposição na orofaringe e maior nos pulmões obtida com o HFA ${ }^{39}$.

Sabe-se que os corticosteróides inalatórios, principalmente em altas doses, podem suprimir o eixo hipotálamohipófise-adrenal. A porcentagem média de alterações na excreção do cortisol em urina de 24 horas de 43 pacientes asmáticos adultos mostra que a dose máxima recomendada de HFA-DPB $640 \mu \mathrm{g} /$ dia produziu a mesma supressão da adrenal que a dose de $672 \mu \mathrm{g} /$ dia de CFC-DPB, considerada intermediária 40 .

A meta-análise conduzida por Lipworth envolvendo quatro estudos que comparam HFA-DPB com CFC-DPB indica que não há supressão da adrenal clinicamente significante quando se usa HFA-DPB em doses menores do que a dose máxima recomendada por dia $(640 \mu \mathrm{g} / \mathrm{dia})^{36}$.

O mesmo resultado foi obtido no estudo conduzido por Pedersen et al., onde 300 crianças de 6 a 11 anos usaram HFADPB e CFC-DPB durante 12 meses, e não foram registradas diferenças significantes no que diz respeito a taxa de crescimento $(p=0,796)$, freqüência de crises de asma $(p=0,517)$, infecções do trato respiratório superior $(p=0,335)$ ou aumento nos sintomas de asma $(p=0,759)^{38}$.

\section{Custo do HFA}

Um ensaio clínico randomizado multicêntrico realizado com grupos de pacientes dos Estados Unidos, Reino Unido, Holanda e Bélgica evidenciaram, após 1 ano de estudo, que o custo em dólares do tratamento de manutenção inalatório por paciente de asma crônica independe do tipo de propelente utilizado (CFC ou HFA), com um custo anual estimado de US\$ 225,62 para o grupo HFA-DPB, que foi ligeiramente menor que o grupo CFC-DPB (US\$321,07). O HFA-DPB apresentou um uma porcentagem de dias sem sintomas maior $(42,4 \%)$ em relação ao grupo CFC-DPB (20\%), com um valor de $p$ estatisticamente significativo $(p=0,006)$. Ao avaliar o custo médio de cada dia sem sintomas, o propelente HFA mostrou ser mais econômico, com um gasto de US\$ 1,36 comparado a um custo de US $\$ 1,81$ para o grupo CFC-DPB. Por isso, os autores concluíram que o controle da asma crônica com HFA teria um custo menor ${ }^{41}$.

Também o grupo liderado por Gross, em trabalho multicêntrico, randomizado e cego com 347 asmáticos adultos, verificou que aqueles em tratamento com HFA-DPB, com a dose de $400 \mu \mathrm{g}$, foram controlados da mesma maneira que os que usaram CFC-DPB a $800 \mu \mathrm{g}$, e concluiu, por conseguinte, que há redução de custos $^{33}$.

É importante salientar que estudos de custo-efetividade do tratamento em asma são escassos e apresentam metodologias variadas, sendo que novos estudos devem ser realizados, especialmente no Brasil.

\section{Período de substituição do CFC pelo HFA e divulgação das informações}

Um fato relevante relacionado à necessidade de substituição do CFC por HFA foi detectado por Donohoe et al. no Reino Unido: a falta de informação sobre essa exigência tanto entre pacientes usuários de IPDM quanto entre profissionais de saúde. 0 estudo evidenciou que $60 \%$ dos pacientes entrevistados não tinham conhecimento do fato, indicando a necessidade de divulgação e esclarecimento em larga escala, uma vez que autoridades britânicas acreditam que a troca do propelente dos IPDM será a maior alteração na composição de medicamentos ocorrida no Reino Unido e poderá resultar em instabilidade no controle da asma, que exigirá atenção especial dos profissionais de saúde ${ }^{42}$. 
Tabela 1 - Principais estudos da eficácia dos inaladores com hidrofluoralcano

\begin{tabular}{|c|c|c|c|c|c|c|}
\hline Autor/País & Droga estudada & Delineamento & $\begin{array}{l}\text { Gravidade } \\
\text { da asma }\end{array}$ & $\begin{array}{l}\text { Tamanho } \\
\text { da amostra }\end{array}$ & $\begin{array}{l}\text { Faixa } \\
\text { etária }\end{array}$ & Conclusão \\
\hline $\begin{array}{l}\text { Gross et al., } \\
\text { EUA }^{33}\end{array}$ & $\begin{array}{l}\text { Dipropionato de } \\
\text { beclometasona }\end{array}$ & $\begin{array}{l}\text { Ensaio clínico } \\
\text { controlado }\end{array}$ & $\begin{array}{l}\text { Leve e } \\
\text { moderada }\end{array}$ & 347 & $18-65$ & $\begin{array}{l}\text { Eficácia do DPB-HFA similar à do } \\
\text { DPB-CFC com metade da dose }\end{array}$ \\
\hline $\begin{array}{l}\text { Farmer et al., } \\
\text { Reino Unido, } \\
\text { África do Sul, } \\
\text { Sérvia } 35\end{array}$ & $\begin{array}{l}\text { Dipropionato de } \\
\text { beclometasona }\end{array}$ & $\begin{array}{l}\text { Ensaio clínico } \\
\text { controlado }\end{array}$ & $\begin{array}{l}\text { Leve e } \\
\text { moderada }\end{array}$ & 229 & $7-12$ & $\begin{array}{l}\text { Eficácia do DPB-HFA } \\
\text { similar à do DPB-CFC } \\
\text { com metade da dose }\end{array}$ \\
\hline $\begin{array}{l}\text { Leach et al., } \\
\text { EUA }^{12}\end{array}$ & $\begin{array}{l}\text { Dipropionato de } \\
\text { beclometasona }\end{array}$ & $\begin{array}{l}\text { Ensaio clínico } \\
\text { controlado }\end{array}$ & Leve & 9 & $18-55$ & $\begin{array}{l}\text { Melhor deposição pulmonar } \\
\text { do DPB-HFA em relação } \\
\text { ao FP-CFC e DPB-CFC }\end{array}$ \\
\hline $\begin{array}{l}\text { Fowler et al., } \\
\text { Reino Unido } 24\end{array}$ & $\begin{array}{l}\text { Propionato de } \\
\text { fluticasona }\end{array}$ & $\begin{array}{l}\text { Ensaio clínico } \\
\text { controlado }\end{array}$ & $\begin{array}{l}\text { Leve e } \\
\text { moderada }\end{array}$ & 18 & $16-70$ & $\begin{array}{l}\text { Eficácia do FP-HFA similar à do } \\
\text { FP-CFC com metade da dose }\end{array}$ \\
\hline $\begin{array}{l}\text { Langley et al., } \\
\text { Reino Unido } 25\end{array}$ & $\begin{array}{l}\text { Propionato de } \\
\text { fluticasona }\end{array}$ & $\begin{array}{l}\text { Ensaio clínico } \\
\text { controlado }\end{array}$ & $\begin{array}{l}\text { Leve e } \\
\text { moderada }\end{array}$ & 59 & $21-41$ & $\begin{array}{l}\text { Eficácia da FP-HFA similar } \\
\text { à da FP em dispositivo de pó }\end{array}$ \\
\hline $\begin{array}{l}\text { Bousquet \& } \\
\text { Cantini, } \\
\text { França } 32\end{array}$ & $\begin{array}{l}\text { Dipropionato de } \\
\text { beclometasona }\end{array}$ & $\begin{array}{l}\text { Ensaio clínico } \\
\text { controlado }\end{array}$ & $\begin{array}{l}\text { Leve e } \\
\text { moderada }\end{array}$ & 1.158 & $15-44$ & $\begin{array}{l}\text { Doses equivalentes de } \\
\text { DPB-HFA e DPB-CFC } \\
\text { facilitam a transição }\end{array}$ \\
\hline $\begin{array}{l}\text { Pedersen et al., } \\
\text { Dinamarca }^{38}\end{array}$ & $\begin{array}{l}\text { Dipropionato de } \\
\text { beclometasona }\end{array}$ & $\begin{array}{l}\text { Ensaio clínico } \\
\text { controlado }\end{array}$ & Leve & 300 & $5-11$ & $\begin{array}{l}\text { Eficácia do DPB-HFA similar à do } \\
\text { DPB-CFC com metade da dose }\end{array}$ \\
\hline $\begin{array}{l}\text { Hauber et al., } \\
\text { Canadá } 26\end{array}$ & Flunisolida & $\begin{array}{l}\text { Ensaio clínico } \\
\text { controlado }\end{array}$ & $\begin{array}{l}\text { Leve e } \\
\text { moderada }\end{array}$ & 12 & $18-50$ & $\begin{array}{l}\text { Supressão de eosinófilos em } \\
\text { vias centrais e periféricas com } \\
\text { melhora da função pulmonar }\end{array}$ \\
\hline $\begin{array}{l}\text { Currie et al., } \\
\text { Reino Unido } 29\end{array}$ & $\begin{array}{l}\text { Fluticasona- } \\
\text { salmeterol }\end{array}$ & $\begin{array}{l}\text { Ensaio clínico } \\
\text { controlado }\end{array}$ & Leve & 14 & $32-40$ & $\begin{array}{l}\text { Eficácia similar entre DPB-HFA } \\
\text { e FLU-HFA com doses iguais }\end{array}$ \\
\hline
\end{tabular}

Hartung et al. relatam os resultados de um inquérito no qual 100 pacientes foram indagados sobre a mudança dos IPDM contendo CFC para inaladores contendo HFA ou para inaladores de pó. Encontraram boa aceitabilidade dos pacientes (96 pacientes), mas alguns preferiram os inaladores de pó (quatro pacientes), ainda que estes apresentem um custo três vezes mais elevado. Finalmente, os autores salientam a importância dos médicos aproveitarem o período de substituição para revisar o tratamento e a técnica inalatória dos pacientes $^{43}$.

\section{Considerações finais}

Novas opções terapêuticas disponibilizadas nos últimos anos revolucionaram o tratamento da asma em todas as idades. Essas novas opções têm em comum a administração pela via inalatória, que permite a otimização da relação eficácia/tolerabilidade no tratamento da asma. As alternativas mais empregadas na administração inalatória de fármacos são os inaladores de pó e os IPDM.

A exigência da troca de propelentes dos IPDM impulsionou a busca de novas terapias para a asma. Os IPDM contendo CFC-DPB resultam em deposição da droga principalmente na orofaringe e levam, por conseguinte, a uma indesejável absorção oral, mas não alcançam adequadamente a inflamação das vias aéreas distais. O HFA-DPB promove maior deposição pulmonar, ainda que a técnica inalatória não seja a ideal, o que permite o uso de uma dose diária menor de corticosteróide. O HFA-DPB é eficaz, bem tolerado e não ocasiona supressão do eixo hipotálamohipófise-adrenal clinicamente significativa nas doses recomendadas.

É importante aproveitar a transição do CFC para o HFA como uma oportunidade para a reavaliação do paciente, levando em conta não só o controle da asma, mas também a qualidade de vida do paciente e a correta utilização da técnica inalatória, para aproveitamento ótimo da medicação.

A maioria das pesquisas realizadas com o HFA emprega o DPB e foi realizada em adultos. Alguns estudos avaliam também o salbutamol, a fluticasona, a flunisolida e a associação fluticasona-salmeterol. Torna-se importante a realização de estudos adicionais avaliando a eficácia e a tolerabilidade dos IPDM-HFA em todas as idades e com os demais fármacos que vinham sendo administrados em associação com o CFC, tais como budesonida, formoterol, brometo de ipratrópio e fenoterol. Os estudos com HFA-DPB podem ser considerados suficientes para a avaliação da tolerabilidade do HFA como propelente, mas não se pode extrapolar com segurança as informações obtidas nesses estudos com relação às vantagens farmacocinéticas identificadas, posto que elas estão relacionadas especificamente com as características físicas das partículas obtidas nos aerossóis de HFA-DPB. No Brasil, as apresentações fluticasona-HFA e fluticasonasalmeterol-HFA já estão disponibilizadas no comércio. Todavia, é importante considerar a escassez de dados na literatura 
acerca do uso do HFA na faixa etária pediátrica, sendo, pois, desejável que fossem conduzidos estudos controlados adicionais em crianças e adolescentes.

\section{Agradecimentos}

Os autores agradecem a Frederico G. Rocha, Gabriela A. Costa e Paula C. A. Silva, estudantes da Faculdade de Medicina da Universidade Federal de Minas Gerais, pela sua contribuição na pesquisa bibliográfica que fundamentou a redação deste artigo.

\section{Referências}

1. Anderson PJ. Delivery options and devices for aerosolized therapeutics. Chest. 2001;120:89-93.

2. Dhand R. Future directions in aerosol therapy. Respir Care Clin N Am. 2001;7:319-35.

3. Szefler S, Warner J, Staab D, Wahn U, Bourgeois M, Zandviliet EEM, et al. Switching from conventional aerosol beclomethasone dipropionate therapy in children: a 6-month, open label, randomized trial. J Allergy Clin Immunol. 2002;110:45-50.

4. Terzano C. Pressurized metered dose inhalers and add-on devices. Pulm Pharmacol Ther. 2001;14:351-66.

5. Montreal Protocol. Substances that deplete the ozone layer. Command Paper n. 977. Treaty Series; 1990.

6. Forte R, Dibble C. The role of international environmental agreements in metered-dose inhaler technology changes. J Allergy Clin Immunol. 1999;104:217-20.

7. Meyer RJ. A United States regulator's perspective on the ongoing chlorofluorocarbon transition. J Allergy Clin Immunol. 1999;104:236-8.

8. Atkins $P$. Chlorofluorocarbon to hydrofluoroalkane formulations: an industry perspective. J Allergy Clin Immunol. 1999;104: 268-70.

9. Tashkin DP. Extra-fine corticosteroid aerosols from hydrofluoroalkane-134a metered-dose inhalers: potential advantages and disadvantages. Chest. 1999;115:316-8.

10. Leach $\mathrm{C}$. Effect of formulation parameters on hydrofluoroalkanebeclomethasone dipropionate drug deposition in humans. J Allergy Clin Immunol. 1999;104:250-2.

11. Brindley A. The chlorofluorocarbon to hydrofluoroalkane transition: the effect on pressurized metered dose inhaler suspension stability. J Allergy Clin Immunol. 1999;104:221-6.

12. Leach $C L$, Davidson PJ, Hasselquist BE, Boudreau RJ. Lung deposition of hydrofluoroalkane-134a beclomethasone is greater than that of chlorofluorocarbon fluticasone and chlorofluorocarbon beclomethasone - A cross-over study in healthy volunteers. Chest. 2002; $122: 510-6$.

13. Janssens $H$, Jongste $J$, Hop W, Tiddens $H$. Extra-fine particles improve lung delivery of inhaled steroids in infants - A study in an upper airway model. Chest. 2003;123:2083-8.

14. Dolovich MB, Fink JB. Aerosols and devices. Respir Care Clin N Am. $2001 ; 7: 131-73$.

15. Borgstrom L. The pharmacokinetics of inhaled hydrofluoroalkane formulations. J Allergy Clin Immunol. 1999;104:246-9.

16. Leach CL. Preclinical safety of propellant HFA-134a and airomir. Br J Clin Pract. 1995;79:10-2.

17. British Asthma Guidelines Coordinating Committee. British guidelines on asthma management: 1995-review and position statement. Thorax. 1997;52:2-24.

18. Leach CL. Enhanced drug delivery through reformulating IPDM with HFA propellants: drug deposition and its effect on pre clinical and clinical programs. In: Dalby RN, Byron R, Farr SJ, editors. Respiratory drug delivery $\mathrm{V}$ proceedings. Buffalo Grove: Interpharm Press; 1996. p. 133-144

19. Hawksworth RJ, Sykes AP, Faris M, Mant T, Lee TH. Albuterol HFA is as effective as albuterol CFC in preventing exercise-induced bronchoconstriction. Ann Allergy Asthma Immunol. 2002;88:473-7.

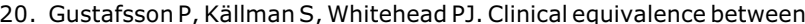
salbutamol hydrofluoroalkane IPDM and salbutamol Turbuhaler the same cumulative microgram doses in paediatric patients. Respir Med. 2002;96:957-9.

21. Langley SJ, Sykes AP, Batty EP, Masterson CM, Woodcock A. A comparison of the efficacy and tolerability of single doses of HFA $134 \mathrm{a}$ albuterol and CFC albuterol in mild-to-moderate asthmatic patients. Ann Allergy Asthma Immunol. 2002;88:488-93.

22. Vondra V, Sladek K, Kotasová J, Terl M, Cantini L. A new HFA-134a propellant in the administration of inhaled DPB via the jet spacer: controlled clinical trial vs the conventional CFC. Respir Med. 2002;96:784-9.
23. Craig-McFeely PM, Wilton LV, Soriano JB, Maier WC, Shakir SAW. Prospective observational cohort safety study to monitor the introduction of a non-CFC formulation of salbutamol with HFA 134 a in England. Int J Clin Pharmacolol Ther. 2003;41:67-76.

24. Fowler SJ, Orr LC, Sims EJ, Wilson AM, Currie GP, McFarlane L, et al. Therapeutic ratio of hydrofluoroalkane and chlorofluorocarbon formulations of fluticasone propionate. Chest. 2002;122:618-23.

25. Langley SJ, Holden J, Derham A, Hedgeland P, Sharma RK, Woodcock A. Fluticasone propionate via the diskhaler or hydrofluoroalkane-134a metered-dose inhaler on methacolineinduced airway yyperresponsiveness. Chest. 2002;122:806-11.

26. Hauber $H$, Gotfried $M$, Newman K, Danda R, Servi RJ, Christodoulopoulos $\mathrm{P}$, et al. Effect of HFA-flunisolide on peripheral lung inflammation in asthma. J Allergy Clin Immunol. 2003; 112:58-63.

27. Nolting A, Sista S, Abramowitz W. Single dose study to compare the pharmacokinetics of HFA flunisolide and CFC flunisolide. J Pharm Sci. 2002; $91: 424-32$

28. Gillman S, Anolik R, Shenkel E, Newman K. One year trial on safety and normal linear growth with flunisolide HFA in children with asthma. Clin Pediatric. 2002;41:333-40.

29. Currie GP, Stenback S, Lipworth BJ. Effects of fluticasne vs. fluticasone/salmeterol on airway calibre and airway hiperresponsiveness in mild persistent asthma. $\mathrm{Br}$ J Clin Pharmacol. 2003;56:11-17

30. Ganderton D, Lewis D, Davier R, Meakin B, Brambilla G, Church T. Modulite: a means of designing the aerosols generated by pressurized metered dose inhalers. Respir Med. 2002;96:3-8.

31. Woodcock A, Acerbi D, Poli G. Moduliteâ technology: pharmacodynamic and pharmacokinetic implications. Respir Med. 2002;96:9-15.

32. Bousquet J, Cantini L. Clinical studies in asthmatics with a new nonextra fine HFA formulation of beclometasone dipropionate (DPB Modulite). Respir Med. 2002;96:17-27.

33. Gross G, Thompson PJ, Chervinsky P, Vanden Burgt J. Hydrofluoroalkane-134a beclomethasone dipropionate, 400 microg, is as effective as chlorofluorocarbon beclomethasone dipropionate, 800 microg, for the treatment of moderate asthma. Chest. 1999; 115:343-51.

34. Magnussen $\mathrm{H}$. Equivalent asthma control after dose reduction with HFA-134a beclomethasone solution aerosol: comparative Inhaled Steroid Investigation Group (CISIG). Respir Med. 2000;94:549-55.

35. Farmer IS, Middle M, Savic J, Perri VL, Herdman MJ. Therapeutic equivalence of inhaled beclomethasone dipropionate with CFC and non-CFC (HFA 134a) propellants both delivered via the Eeasibreathe inhaler for the treatment of paediatric asthma. Respir Med. 2000;94:57-63.

36. Lipworth BJ. The comparative safety/efficacy ratio of HFA-DPB. Respir Med. 2000;94:21-6.

37. Cruz AA, Ponte EV. Inflamação nas pequenas vias aéreas em asmáticos. Rev Bras Alerg Imunupatol. 2003;26:25-32.

38. Pedersen S, Warner J, Wahn U, Staab D, Le Bourgeois M, Van Essen-Zandvliet $E$, et al. Growth, systemic safety, and efficacy during 1 year of asthma treatment with different beclomethasone dipropionate formulations: an open-label, randomized comparison of extrafine and conventional aerosols in children. Pediatrics. 2002;109:1-10.

39. Vanden Burgt JA, Busse WW, Martin RJ, Szefler SJ, Donnell D. Efficacy and safety overview of a new inhaled corticosteroid, OVAR (hydrofluoroalkane-beclomethasone extrafine inhalation aerosol), in asthma. J Allergy Clin Immunol. 2000;106:1209-26.

40. Harrison LI, Colice GL, Donnell D, Soria I, Dockhorn R. Adrenal effects and pharmacokinetics of CFC-free beclomethasone dipropionate: a 14-day dose-response study. J Pharm Pharmacol. 1999;51:263-9.

41. Price D, Haughney J, Duerden M, Nicholls C, Moseley C. The cost effectiveness of chlorofluorocarbon-free beclomethasone dipropionate in the treatment of chronic asthma: a cost model based on a 1-year pragmatic, randomised clinical study. Pharmacoeconomics 2002; 20:653-64.

42. Donohoe $H$. Preparing patients and health professionals for the transition to chlorofluorocarbon-free inhalers: the British perspective. J Allergy Clin Immunol. 1999;104:239-42.

43. Hartung TK, Allbutt $H$, Dewar M, Innes JA, Cromptom GK. Moving from CFC aerosol to HFA aerosol or dry powder inhalers: What do patients think? Respiration. 2002;69:314-9.

Correspondência:

Paulo Augusto Moreira Camargos

Departamento de Pediatria, Faculdade de Medicina, UFMG

Av. Professor Alfredo Balena, 190/4061

CEP 30130-100 - Belo Horizonte, MG

Fone: (31) 3248.9772 - Fax: (31) 3248.9664

E-mail: pcamargs@medicina.ufmg.br 